\title{
Reflets
}

Revue d'intervention sociale et communautaire

\section{Ressources mobilisées par des femmes arabo-musulmanes victimes de violence conjugale en contexte migratoire}

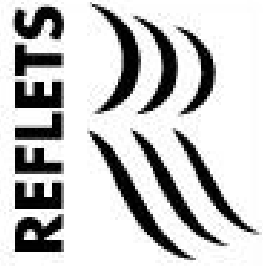

\section{Bouchra Taïbi}

Volume 21, numéro 1, printemps 2015

Violences faites aux femmes et contextes minoritaires

URI : https://id.erudit.org/iderudit/1032552ar

DOI : https://doi.org/10.7202/1032552ar

Aller au sommaire du numéro

Éditeur(s)

Reflets, Revue d'intervention sociale et communautaire

ISSN

1203-4576 (imprimé)

1712-8498 (numérique)

Découvrir la revue

Citer cet article

Taïbi, B. (2015). Ressources mobilisées par des femmes arabo-musulmanes victimes de violence conjugale en contexte migratoire. Reflets, 21(1), 161-188. https://doi.org/10.7202/1032552ar

\section{Résumé de l'article}

Le présent article s'intéresse aux femmes arabes et musulmanes victimes de violence conjugale en contexte migratoire. Il a pour but de présenter les ressources qu'elles mobilisent tout en mettant en évidence le contexte avec lequel elles doivent composer pour faire face à leur situation. Il s'appuie sur une étude qualitative exploratoire menée auprès de quatre femmes nées au Maghreb et résidant au Québec. L'étude met en lumière que les stratégies déployées et les ressources mobilisées par les femmes sont étroitement liées d'une part à leur désir de s'insérer sur le marché de l'emploi et d'autre part au fait qu'elles craignent que la décision de se séparer de leur conjoint entraîne pour elles un renvoi dans leur pays d'origine.
Tous droits réservés (C) Reflets, Revue d'intervention sociale et communautaire, 2015
Ce document est protégé par la loi sur le droit d'auteur. L'utilisation des services d'Érudit (y compris la reproduction) est assujettie à sa politique d'utilisation que vous pouvez consulter en ligne. 


\section{Ressources mobilisées par des femmes arabo-musulmanes victimes de violence conjugale en contexte migratoire}

\section{Bouchra Taïbi}

Étudiante au doctorat en travail social et chargée de cours, École de travail social, UQAM

\section{Résumé}

Le présent article s'intéresse aux femmes arabes et musulmanes victimes de violence conjugale en contexte migratoire. Il a pour but de présenter les ressources qu'elles mobilisent tout en mettant en évidence le contexte avec lequel elles doivent composer pour faire face à leur situation. Il s'appuie sur une étude qualitative exploratoire menée auprès de quatre femmes nées au Maghreb et résidant au Québec. L'étude met en lumière que les stratégies déployées et les ressources mobilisées par les femmes sont étroitement liées d'une part à leur désir de s'insérer sur le marché de l'emploi et d'autre part au fait qu'elles craignent que la décision de se séparer de leur conjoint entraîne pour elles un renvoi dans leur pays d'origine.

Mots clés : femmes arabes et musulmanes, femmes immigrantes, violence conjugale, intersectionnalité, ressources mobilisées, stratégies de recherche d'aide 


\section{Abstract}

This article focuses on Arab and Muslim women victims of domestic violence in the context of migration. It aims to present the resources they mobilize and the context they face when dealing with their situation. It is based on an exploratory qualitative study of four women born in North Africa and residing in Quebec. The study highlights that the strategies and resources mobilized by women are closely related, firstly, to their desire to work and, secondly, the fear to be sent back to their country of origin if they separate from their spouse.

Key words: Arab and Muslim women, immigrant women, domestic violence, intersectionality, resources mobilized, help seeking strategy

\section{Introduction}

Le présent article expose les résultats d'une étude menée dans le cadre d'un mémoire de maitrise en travail social ${ }^{1}$ portant sur les expériences de violence conjugale de femmes arabo-musulmanes établies au Québec et les ressources qu'elles mobilisent pour faire face à leur situation ${ }^{2}$. Appréhendée à partir d'un cadre théorique basé sur l'intersectionnalité, cette étude visait d'une part à comprendre de quelle manière la position sociale de ces femmes, à l'intersection du genre et de la race, structure leur expérience de la violence conjugale et d'autre part, à examiner comment elles font face à leur situation. Les trajectoires de quatre femmes maghrébines, depuis la rencontre avec leur conjoint jusqu'à la séparation du couple, ont été saisies par le biais d'entrevues semi-directives en nous inspirant de la méthode de récit de vie. Cette méthode a permis de situer le contexte dans lequel s'est inscrite l'expérience de violence conjugale des répondantes. Dans l'article, nous nous intéressons plus particulièrement aux ressources mobilisées par les femmes. Nous débuterons par une revue de la littérature sur les violences auxquelles les femmes arabes 
et musulmanes sont exposées dans l'espace public afin de situer le contexte avec lequel elles doivent composer lorsqu'elles sont victimes de violence conjugale. Suivra un état des connaissances sur l'utilisation des services d'aide en violence conjugale par les femmes arabes et musulmanes. Nous exposerons ensuite sommairement la méthodologie de recherche utilisée. Enfin, nous présenterons les principales ressources qui ont été mobilisées par les répondantes tout au long de leur trajectoire.

\section{Femmes arabes et musulmanes et violence dans l'espace public : état des lieux}

«La situation des femmes arabes et musulmanes au Québec se caractérise notamment par les violences qui sont perpétrées à leur encontre dans l'espace public. »
Au Québec, malgré l'abondance de la littérature scientifique sur la violence faite aux femmes, peu d'écrits portent sur la situation spécifique des femmes arabes et musulmanes. Néanmoins, les quelques auteures ou auteurs qui s'y sont intéressés s'accordent pour dire que "certaines de leurs situations sont similaires et communes à celles vécues par les autres femmes immigrantes " (Osmani, 2007, p. 6), mais que d'autres leur sont spécifiques compte tenu du climat anti-arabo-musulman qui prévaut (AbuRas, 2007; Bendriss, 2005; 2007; 2009; Chouakri, 2003; Osmani, 2007).

La situation des femmes arabes et musulmanes au Québec se caractérise notamment par les violences qui sont perpétrées à leur encontre dans l'espace public. À cet effet, la littérature mentionne le sentiment d'insécurité et d'hostilité qu'elles éprouvent, le harcèlement racial qu'elles subissent - surtout en milieu de travail - et plus généralement leur sentiment d'exclusion du marché du travail (Bendriss, 2009; Chouakri, 2003; Osmani, 2007). Plusieurs soutiennent que ce sentiment d'exclusion est ressenti plus durement par celles qui portent le voile (Bendriss, 2009; Osmani, 2007; Bouchard et Taylor, 2008; Vashti Persad et Lukas, 2002). À cet effet, une étude menée auprès de Canadiennes musulmanes qui portaient le voile pendant leur recherche d'emploi dans les secteurs manufacturiers, de la vente et des services révèle que $40,6 \%$ des répondantes se sont fait dire par un employeur que 
si elles souhaitaient obtenir l'emploi convoité, elles devaient ôter leur voile (Vashti Persad et Lukas, 2002). Enfin, Bendriss (2009) souligne qu'outre le fait que les femmes arabes et musulmanes partagent avec l'ensemble des femmes immigrantes la difficulté de faire reconnaitre leurs diplômes obtenus à l'étranger et leurs expériences antérieures de travail, les perceptions particulièrement négatives à leur encontre augmentent leurs difficultés d'insertion sur le marché du travail en particulier et dans la société en général.

Nombreux sont les écrits en violence conjugale qui soutiennent qu'une situation socioéconomique défavorable peut limiter la capacité des femmes à être autonomes et engendrer une dépendance au conjoint. Il est difficile d'établir un portrait de la situation socioéconomique des femmes arabes et musulmanes, car les données disponibles sont rarement différenciées à la fois par le sexe, l'origine ethnique et la confession religieuse des personnes. Néanmoins, une exploration de différentes sources nous permettent de dégager que de façon générale, les femmes arabes et musulmanes forment un groupe socioéconomique défavorisé, et ce, malgré leur haut niveau d'instruction et de mâtrise de la langue, française ou anglaise. À titre indicatif, $25 \%$ des Canadiennes d'origine arabe détiennent un diplôme universitaire, soit un taux plus élevé que celui observé dans la population canadienne en général qui est de $15 \%$ (Statistique Canada, 2007). Parmi les Canadiennes musulmanes de toutes origines confondues, près du tiers détiennent un diplôme universitaire (Hamdani, 2004). Malgré cela, leur taux de chômage est deux fois plus élevé comparativement à l'ensemble des Canadiennes (Hamdani, 2006, p. 13). En ce qui a trait au revenu, en 2000,16\% de la population canadienne totale avait des revenus inférieurs au seuil de faible revenu, alors que c'était le cas pour plus du double (35\%) des personnes qui ont fait état d'une ascendance arabe (Statistique Canada, 2007).

Parmi les autres discriminations qui affectent particulièrement les femmes arabes et musulmanes, Chouakri (2003) et Osmani (2007) soulignent le sexisme et le racisme de la politique canadienne d'immigration. Chouakri (2003) montre que malgré la féminisation mondiale de l'immigration vers le Canada et le 
"...le parrainage

provoque une

dépendance vis-à-vis

du conjoint, voire

"constitue un outil de

choix pour perpétuer

l'abus »."
Québec et l'augmentation de l'immigration originaire de pays arabes et musulmans, les femmes arabes et musulmanes sont moins nombreuses à pouvoir immigrer ou à être reçues comme requérantes principales. Ainsi, tout comme Chouakri (2003), Osmani $(2007$, p. 25$)$ note qu'à défaut de pouvoir se qualifier comme requérantes principales, les femmes originaires des pays arabes et musulmans "sont majoritaires dans la catégorie des personnes parrainées comparativement aux hommes ». À ce sujet, Côté, Kérisit et Côté (2001, p. 67) comptent parmi les nombreuses auteures qui soutiennent que le parrainage provoque une dépendance vis-à-vis du conjoint, voire « constitue un outil de choix pour perpétuer l'abus".

\section{Les services d'aide utilisés par les femmes arabes et musulmanes victimes de violence conjugale : état les lieux}

Nous l'avons souligné, peu d'études portent spécifiquement sur les femmes arabes et musulmanes victimes de violence conjugale. Néanmoins, quelques recherches se sont intéressées aux types de service d'aide qu'elles utilisent. Réalisée par Saris et collab. (2007), l'une d'elles examine les ressources auxquelles recourent les Canadiennes musulmanes pour résoudre les conflits familiaux ainsi que les raisons de leurs choix. Pour ce faire, 37 intervenantes ou intervenants ont été rencontrés et des entretiens semi-directifs ont été réalisés auprès de 24 Canadiennes musulmanes, dont 18 ont vécu des conflits familiaux. Parmi ces dernières, 9 ont déclaré avoir vécu une violence conjugale.

En ce qui concerne les buts recherchés par les Canadiennes musulmanes vivant un conflit familial, Saris et collab. (2007, p. 30) notent que la détermination à maintenir la relation familiale et l'appréhension quant au statut de divorcée poussent certaines femmes « à opter pour des solutions plus conciliantes et moins radicales de rupture, comme la séparation de corps qui, dans leur compréhension, préserve le lien familial ». Toutefois, les auteurs 
soutiennent que "l'intention exprimée par les Canadiennes musulmanes interviewées, à préserver le mariage et à considérer le divorce comme ultime solution, tire sa force d'autres facteurs " (p. 31) qui sont essentiellement la crainte du regard d'autrui en général, la crainte de décevoir une personne en particulier (par exemple, les parents) ou la crainte de perdre leurs enfants.

Toujours selon Saris et collab. (2007), des critères bien précis incitent les Canadiennes musulmanes à opérer un véritable magasinage entre les différentes ressources disponibles pour régler leurs conflits familiaux : la proximité géographique, spirituelle, culturelle ou linguistique de l'intervenante ou de l'intervenant, sa disponibilité, le partage des valeurs, le coût, la confidentialité et la sécurité (p. 46). Parmi les 18 répondantes qui ont été confrontées à un conflit familial, 6 seulement ont sollicité l'aide d'un conseiller religieux. Le système judiciaire (11/18), les avocates et avocats $(13 / 18)$, les travailleuses et les travailleurs sociaux et communautaires $(9 / 18)$ et les services de la police ou de la Direction de la Protection de la Jeunesse (6/18) ont par contre fait l'objet de fréquents recours (p. 55). Les demandes des répondantes vis-à-vis des intervenantes et des intervenants ont pris trois formes : 1 . demandes d'information sur des questions légales (comme le droit québécois et canadien concernant la garde des enfants) ou religieuses (comme les préceptes régissant la séparation ou le divorce); 2 . demandes d'intervention auprès du mari;3. demandes de soutien psychologique qui comprennent autant l'écoute que l'accompagnement. Ainsi, les auteurs de l'étude observent que les Canadiennes musulmanes « s'adressent de manière simultanée, successive ou occasionnelle, vers toute personne qui pourrait les aider à trouver un moyen de résoudre les problèmes conjugaux auxquels elles font face " (p. 55).

Une autre étude a été réalisée aux États-Unis par Abu-Ras (2007) auprès de femmes arabes $(n=67)$ qui ont été victimes de violence conjugale et qui ont utilisé les services offerts par un organisme situé au Michigan. Celui-ci offre des services d'aide générale à la communauté arabe, dont certains spécifiques à la violence conjugale. En ce qui concerne les services spécifiquement destinés aux femmes victimes de violence conjugale, l'auteure 
note qu'une faible proportion de femmes a fait appel à la ligne d'aide ou utilisé les services d'hébergement (8\%). Celles qui ont sollicité un service d'hébergement pour obtenir une assistance constituent également un faible pourcentage (18\%). Par contre, les femmes sont beaucoup plus nombreuses à avoir utilisé les services de consultation familiale (70\%) et d'aide juridique (52\%). AbuRas (2007) croit que la faible utilisation des services pourrait être due d'une part au fait qu'ils accusent certaines faiblesses (manque d'équipement, hébergement éloigné, ligne d'urgence plus ou moins fiable) et d'autre part à la crainte qu'ont ces femmes victimes de violence conjugale d'être stigmatisées par leur communauté et leur famille si elles sollicitent de tels services. Quant à la plus grande utilisation des services généraux (consultation familiale et aide légale), l'auteure croit que cela est imputable au fait qu'ils sont offerts gratuitement et en arabe et que l'organisme est davantage connu pour les services généraux qu'il offre (aide à l'emploi, cours d'anglais, services médicaux, aide légale, etc.) que pour ceux d'aide en violence conjugale.

Dans une autre étude réalisée auprès de membres de la communauté arabo-musulmane de London en Ontario, Baobaid (2002) s'est intéressé aux barrières empêchant les femmes d'utiliser les services d'aide en violence conjugale. Pour ce faire, il s'est basé sur les témoignages recueillis lors de discussions de groupes et d'entrevues individuelles menées auprès de femmes et d'hommes arabo-musulmans. Du point de vue des répondantes et des répondants, les femmes arabo-musulmanes ont peu tendance à recourir auxdits services et lorsqu'elles le font, c'est longtemps après avoir sollicité le soutien de leurs réseaux familiaux et communautaires.

Des auteures ou auteurs (Abugideiri, 2007; Alkhateeb et Abugideiri, 2007; Abu-Ras, 2007; Baobaid, 2002; Burman et Chantler, 2005; Haj-Yahia, 2002; Haj-Yahia et Sadan, 2008;West, 2005) soulignent en grand nombre que la crainte d'être critiquées par la communauté d'appartenance et la famille, pour plusieurs raisons différentes évoquées, peut dissuader les femmes ethnicisées ou racisées à utiliser les services d'aide. 
"...les femmes hésitent à demander de l'aide parce qu'elles craignent de perdre le respect de leur entourage de même que leur statut au sein de leur communauté et de leur famille..."
Dans les écrits qui portent spécifiquement sur les femmes arabes et musulmanes, une première interprétation est d'ordre culturel et repose sur l'idée que pour les Arabo-musulmans faire intervenir des personnes étrangères dans les affaires familiales est une atteinte aux valeurs du mariage. Dans la perspective de cette interprétation, les femmes hésitent à demander de l'aide parce qu'elles craignent de perdre le respect de leur entourage de même que leur statut au sein de leur communauté et de leur famille (Abu-Ras, 2007; Baobaid, 2002; Haj-Yahia, 2002; Haj-Yahia et Sadan, 2008). Toujours selon cette perspective culturaliste, les femmes sont éduquées à être conciliantes et obéissantes face à leur conjoint, malgré la violence existante, parce que l'harmonie et l'unité familiale priment sur le bien-être individuel (Haj-Yahia, 2002; Haj-Yahia et Sadan, 2008). Cette perspective semble orienter la plupart des études que nous avons relevées, notamment celles de Abugideiri (2007), d'Abu-Ras (2007), de Baobaid (2002), de Haj-Yahia (2002) et de Haj-Yahia et Sadan (2008), et qui portent spécifiquement sur les femmes d'origine arabe ou de confession musulmane qui sont victimes de violence conjugale. Ces travaux examinent la manière dont les croyances culturelles, dites "traditionnelles", des femmes influencent leurs perceptions de la violence conjugale et leurs habitudes d'utilisation des services d'aide. Globalement, nous pouvons dire que l'idée qui ressort de cette école de pensée est que plus les femmes adhèrent aux valeurs traditionnelles, moins elles ont tendance à solliciter les services d'aide en violence conjugale.

Selon une deuxième interprétation, si les femmes qui sollicitent les services d'aide en violence conjugale sont critiquées par leurs communautés d'appartenance ou par leur famille, c'est parce ces dernières craignent que cela renforce la représentation dominante que l'on a des groupes arabes et musulmans, à savoir qu'ils sont plus violents que tout autre groupe (Alkhateeb et Abugideiri, 2007; Bendriss, 2007).

On trouve dans les écrits d'Abu-Ras (2007) et de Bendriss (2007) une troisième interprétation quant aux raisons qui peuvent dissuader les femmes de recourir aux services d'aide compte tenu du climat anti-arabo-musulman. Abu-Ras (2007) note 
"...le climat qui prévaut depuis le 11 septembre et les changements opérés dans la politique d'immigration font que les femmes arabes victimes de violence conjugale redoutent d'alerter le système judiciaire de crainte que leur famille soit davantage l'objet de harcèlement ou que leur conjoint soit déporté ou maltraité en raison de son origine. " qu'au moment de réaliser son étude, soit avant le 11 septembre 2001, 58 \% des répondantes estimaient que la meilleure façon de gérer une situation de violence conjugale est de faire arrêter son conjoint (p. 1022). Cependant, l'auteure observe que le climat qui prévaut depuis le 11 septembre et les changements opérés dans la politique d'immigration font que les femmes arabes victimes de violence conjugale redoutent d'alerter le système judiciaire de crainte que leur famille soit davantage l'objet de harcèlement ou que leur conjoint soit déporté ou maltraité en raison de son origine. Abu-Ras (2007) soutient que le climat anti-arabe qui sévit aux États-Unis ainsi que les perceptions négatives à l'égard de la communauté arabe dissuadent plusieurs femmes d'utiliser les services d'aide disponibles et cela, notamment parce qu'elles veulent protéger leur famille. Pour sa part, Bendriss $(2007$, p. 9) suggère que du fait qu'elles sont conscientes des stéréotypes négatifs à leur encontre et à l'encontre des membres de leurs groupes d'appartenance, certaines femmes arabes et musulmanes victimes de violence conjugale "peuvent choisir de ne pas recourir aux services d'aide offerts par les associations et institutions de la société majoritaire par crainte précisément de renforcer davantage ceux-ci».

\section{Aspects méthodologiques}

Notre revue de la littérature nous a permis de constater que la voix des principales concernées a peu été entendue. De plus, les ressources utilisées par les femmes arabes et musulmanes pour faire face à leur situation ont le plus souvent été explorées à partir d'un examen de leurs habitudes d'utilisation des services d'aide spécifique à la violence conjugale, occultant ainsi d'autres types de ressources qu'elles auraient pu mobiliser. Notons par ailleurs que dans un contexte où les représentations sociales des femmes arabes et musulmanes sont aujourd'hui plus que jamais particulièrement négatives (Bendriss, 2005; 2007; 2009), le fait que les ressources utilisées par celles qui sont victimes de violence conjugale soient peu documentées et que leurs voix soient peu 
"C'est avec le dessein de faire contrepoids à la vision dominante, quoique non hégémonique, par laquelle sont caractérisées les femmes arabes et musulmanes que nous avons voulu leur donner la parole et vous les présenter dans leur rôle d'actrices sociales. " entendues contribue à maintenir le stéréotype de la femme victime, sans voix et sans pouvoir.

C'est avec le dessein de faire contrepoids à la vision dominante, quoique non hégémonique, par laquelle sont caractérisées les femmes arabes et musulmanes que nous avons voulu leur donner la parole et vous les présenter dans leur rôle d'actrices sociales. Pour ce faire, nous avons rencontré quatre femmes d'origine maghrébine âgées de 27 à 52 ans qui ont vécu une expérience de violence conjugale au Canada. Leur trajectoire de vie, depuis leur engagement conjugal jusqu'à la séparation du couple, a été reconstituée à partir d'entretiens semi-directifs et selon la méthode du récit de vie. Chaque entretien a duré de deux à trois heures. Le canevas d'entrevue comportait des questions portant sur les thèmes suivants : les circonstances entourant la rencontre du conjoint; la trajectoire migratoire; les violences vécues; les réactions de la femme face à la violence et les ressources sollicitées; sa perception à propos de la prévalence de la violence conjugale au sein des communautés arabes et musulmanes; et le regard qu'elle pose sur son expérience avec le recul. Le tableau suivant présente les caractéristiques des quatre femmes qui constituent l'échantillon de l'étude. Certaines informations, entre autres, les noms des participantes, ont été modifiées afin de préserver leur anonymat.

Caractéristiques des femmes constituant l'échantillon

\begin{tabular}{|l|c|c|c|c|}
\hline & Hayat & Nora & Hakima & Amira \\
\hline Âge & 52 ans & 27 ans & 29 ans & 33 ans \\
\hline Nombre d'enfants & 1 & 0 & 0 & 0 \\
\hline Niveau d'étude & $\leq$ Élémentaire & Universitaire & Universitaire & $\geq$ Collégial \\
\hline $\begin{array}{l}\text { Statut d'immigration à } \\
\text { l'arrivée au Canada }\end{array}$ & $\begin{array}{c}\text { Résidente } \\
\text { permanent } \\
\text { (parrainée) }\end{array}$ & $\begin{array}{c}\text { Résidente } \\
\text { permanent } \\
\text { (parrainée) }\end{array}$ & $\begin{array}{c}\text { Résidente } \\
\text { permanent } \\
\text { (parrainée) }\end{array}$ & $\begin{array}{c}\text { Résidente } \\
\text { temporaire }\end{array}$ \\
\hline $\begin{array}{l}\text { Durée de cohabitation en } \\
\text { couple }\end{array}$ & $>30$ ans & 6 semaines & 1 mois & 22 mois \\
\hline $\begin{array}{l}\text { Durée d'établissement au } \\
\text { Canada }\end{array}$ & 10 ans & 4 mois & 5 mois & 30 mois \\
\hline
\end{tabular}


Les entrevues ont été retranscrites puis codées. L'analyse du contenu a été effectuée en adoptant la perspective de l'intersectionnalité (Crenshaw, 2005). L'intersectionnalité est une approche qui étudie comment le positionnement des femmes racisées/ethnicisées, à l'intersection de la race et du genre, « rend leur expérience concrète de la violence conjugale, du viol et des mesures pour y remédier qualitativement différente de celles des femmes blanches » (Crenshaw, 2005, p.51). Nous avons considéré l'intersectionnalité comme une lunette facilitant la mise en lumière de la relation entre les violences auxquelles sont exposées les femmes arabes et musulmanes dans l'espace public et la violence conjugale qu'elles subissent dans l'espace privé. Il s'agit d'un cadre qui donne la possibilité de reconnaître ces formes de violence comme étant des obstacles qui peuvent dissuader les femmes de mettre fin à une relation abusive ou encore limiter les ressources auxquelles elles ont accès pour le faire.

\section{Les ressources mobilisées par les femmes : diversité et complémentarité}

En nous intéressant à la trajectoire de vie des femmes depuis leur rencontre avec leur conjoint jusqu'à leur séparation, nous avons pu recueillir des informations tant sur leur vécu de violence que sur les ressources qu'elles ont mobilisées pour faire face à leur situation. Cela nous a permis non seulement de répertorier lesdites ressources, mais également de connaitre le moment où elles ont été utilisées, comment elles l'ont été et à quelle fin, de même que la manière dont elles ont pu influencer l'utilisation ou la non-utilisation d'autres ressources.

\section{Le réseau familial transnational : «Je ne voulais pas inquiéter ma famille."}

Aucune des répondantes de notre étude n'avait de parenté ici au Canada. Néanmoins, toutes se sont organisées pour maintenir des contacts réguliers avec leurs familles restées au pays (par 
téléphone, courriel, Skype), et ce, malgré le fait que presque tous les conjoints $(n=3)$ ont tenté de contrôler la fréquence ou encore le contenu des échanges qu'elles avaient avec elles ${ }^{3}$. Mais malgré ces contacts réguliers, les quatre répondantes ont exprimé ne pas avoir voulu parler dès le départ de la violence qu'elles subissaient parce qu'elles ne souhaitaient pas que leurs familles s'inquiètent pour elles. Ainsi, les résultats de notre étude indiquent que les femmes ont décidé de lui révéler la violence qu'elles subissaient bien après avoir sollicité $d$ 'autres ressources $(n=3)$ ou encore, après que le conjoint ait lui-même impliqué la famille et la belle-famille dans les disputes du couple $(\mathrm{n}=1)$.

Toutes les femmes affirment que les contacts qu'elles ont maintenus avec leurs familles outre-mer leur ont permis de ne pas se sentir complètement isolées et d'avoir accès à du soutien que trois d'entre elles ont qualifié comme ayant été important. Ce soutien était essentiellement psychologique (écoute, conseils, encouragements et appui), mais aussi financier (proposition de transférer de l'argent ou de payer un billet d'avion pour permettre à la femme de revenir au pays). Les familles de deux répondantes ont aussi offert d'intervenir auprès du conjoint, mais les femmes ont jugé qu'une telle intervention risquait d'aggraver la situation, car il valait mieux que le conjoint ne sache pas qu'elles s'étaient confiées à elles.

Ainsi, soucieuses de ne pas inquiéter leurs familles, mais aussi conscientes de l'aide limitée que celles-ci pourraient apporter en raison de la distance, c'est d'abord à des personnes vivant ici que les répondantes se sont confiées.

\section{Le réseau social développé par les femmes ici : une source de soutien et $d^{\prime}$ 'information}

Pendant la durée de leur relation conjugale, toutes les femmes, sauf une, ont réussi malgré la violence subie et leur arrivée récente au pays à développer un certain réseau social. L'étendue de ce réseau est évidemment étroitement liée à la possibilité qu'elles avaient de sortir et de se déplacer sans leur conjoint, autrement dit, d'échapper au contrôle qu'il exerçait. Néanmoins, les données 
"...les femmes ont fait preuve de créativité en utilisant à profit les quelques possibilités qu'elles avaient, aussi minimes soient-elles, pour socialiser, se confier, obtenir des conseils, s'informer de leurs droits et des ressources existantes. » de l'étude indiquent que les femmes ont fait preuve de créativité en utilisant à profit les quelques possibilités qu'elles avaient, aussi minimes soient-elles, pour socialiser, se confier, obtenir des conseils, s'informer de leurs droits et des ressources existantes. Par exemple, une répondante dont le conjoint ne lui fournissait pas l'argent nécessaire pour utiliser les transports en commun devait marcher chaque jour une longue distance pour se rendre aux ateliers d'intégration destinés aux nouveaux arrivants. Elle profitait de cette sortie pour s'arrêter à un parc fréquenté par des personnes de même origine et de même religion qu'elle; ces personnes l'ont alertée sur le cas de femmes maghrébines ayant vécu une situation similaire à la sienne et dont le conjoint s'est organisé pour - ou a tenté de - les renvoyer au pays. Ces mêmes personnes ont alerté la répondante sur le fait que certains hommes sont des voyous et lui ont conseillé d'être vigilante, par exemple, en ayant toujours avec elle ses bijoux, son argent et ses pièces d'identité ou en ouvrant un compte bancaire à son nom seulement afin que ses parents puissent lui transférer de l'argent sans que son conjoint le sache. De même, elles l'ont encouragée à prier pour garder espoir et avoir l'aide de Dieu quoiqu'il advienne. Cette répondante a également profité des ateliers d'intégration pour se lier d'amitié avec des femmes qui l'ont encouragée à demander de l'aide et qui l'ont conduite dans un centre de femmes pour qu'elle y rencontre une intervenante. Une autre répondante profitait de ses sorties à la mosquée le vendredi, jour de la grande prière, pour se changer les idées en socialisant avec d'autres femmes, mais aussi pour consulter l'imam. Elle profitait également des sorties de son mari pour se confier à sa voisine immédiate avec qui elle se sentait confortable parce qu'elle aussi était immigrante, précise-t-elle.

En bref, on note que les femmes se sont entourées de personnes qui leur ont permis notamment de s'informer sur leurs droits et sur les ressources disponibles. 


\section{Les démarches d'insertion socioprofessionnelle : une occasion de s'informer sur les droits et un tremplin pour utiliser des services d'aide en violence conjugale}

Tel que nous l'avons évoqué plus tôt, quelques recherches portant sur les femmes arabes et musulmanes suggèrent que celles-ci auraient tendance à recourir aux services d'aide en violence conjugale longtemps après avoir sollicité le soutien de leurs réseaux familiaux et communautaires (Abu-Ras, 2007; Baobaid, 2002; Haj-Yahia, 2002; Haj-Yahia et Sadan, 2008). S'il est vrai que les services d'aide spécialisée en violence conjugale n'ont pas été sollicités en premier lieu par les répondantes de notre étude, on note toutefois que les trois répondantes arrivées récemment ont entrepris dès leur arrivée au Canada, et malgré la résistance de leur conjoint, des démarches visant leur insertion socioprofessionnelle. En outre, elles ont fait des recherches sur les procédures à suivre pour faire reconnaitre leurs diplômes $(n=2)$ ainsi que leur permis de conduire $(n=2)$. Elles se sont également informées - ou du moins ont tenté d'obtenir de l'information — sur les programmes d'études existants, les conditions d'admission et les frais de scolarité $(n=3)$. Aussi, elles ont contacté au moins un organisme pour s'informer sur les services d'aide en employabilité qui y sont offerts $(n=3)$. Parmi ces organismes, on compte une association d'aide pour l'intégration des immigrants, un centre local d'emploi et des centres de femmes.

Certes, ces démarches ne visaient pas à résoudre spécifiquement leur problème de violence conjugale. Cependant, cela pourrait suggérer que les répondantes s'organisaient pour disposer d'une certaine autonomie qui en retour les sortirait du rapport de dépendance dans lequel elles se trouvaient en raison de leur statut de femme parrainée.

Par ailleurs, les répondantes ont exprimé qu'au moment où elles sont arrivées au Canada, elles ne savaient pas si compte tenu de leur statut elles avaient le droit de travailler ou d'étudier; et 
c'est précisément dans le but de s'informer à ce sujet qu'elles se sont adressées à des organismes offrant des services d'aide en employabilité. Ainsi, Nora et Hakima qui avaient leur résidence permanente y ont appris qu'elles avaient le droit de travailler ou d'étudier, et qu'elles pouvaient aussi être aidées pour leur situation de violence conjugale. Quant à Amira, qui est arrivée au Canada avec un statut de résidente temporaire, elle a appris qu'elle ne pourrait travailler sans obtenir au préalable un permis de travail ou sa résidence permanente, ce dont elle a tenu compte avant de quitter son conjoint. Situation éloquente, le jour où elle s'est retrouvée à l'hôpital, elle aurait voulu y demander de l'aide pour la violence qu'elle subissait, mais elle s'est toutefois résignée à retourner à la maison avec son conjoint :

«C'est comme je disais même si ok je le quitte ou quoi que ce soit, je peux pas travailler parce que j'ai pas ma résidence, je peux rien faire, je peux pas...euh partir à l'hôpital voir un médecin ou faire un suivi ou... [silence)." (Amira)

Cette répondante a donc attendu d'obtenir sa résidence permanente avant de quitter son conjoint.

Nora, qui avait déjà sa résidence permanente en arrivant au Canada, a aussi contacté peu après son arrivée un organisme offrant des services d'aide à l'emploi. Mais alors qu'elle avait répertorié cet organisme en cherchant des ressources qui pourraient l'informer sur les démarches à suivre pour travailler ou étudier, c'est vers ce même organisme qu'elle s'est tournée lorsqu'elle a été frappée par son conjoint et abandonnée seule à la maison. Après lui avoir payé un taxi, l'organisme en question l'a dirigée vers une maison d'hébergement; Nora a immédiatement déposé une plainte à la police pour la violence physique qu'elle avait subie. Comme elle le dit elle-même : «Dieu a voulu que j'utilise ces renseignements-là pour d'autres motifs que le travail. "

En bref, le désir d'occuper un emploi et d'étudier a spontanément amené les répondantes à s'adresser à des organismes offrant des services d'aide à l'emploi. Par ce biais, elles ont pu obtenir une panoplie d'informations dont elles se sont servies, 
" ...organismes offrant des services d'aide en employabilité se sont avérés être de véritables tremplins pour l'accès à d'autres services, dont ceux offerts par les maisons d’hébergement. " notamment pour obtenir des services d'aide en situation de violence conjugale. Ainsi, il est intéressant de constater que les organismes offrant des services d'aide en employabilité se sont avérés être de véritables tremplins pour l'accès à d'autres services, dont ceux offerts par les maisons d'hébergement.

\section{Les maisons d'hébergement : des services grandement appréciés, mais une durée de séjour qui semble trop courte pour les femmes}

À la suite de leur séparation, les trois répondantes d'arrivée récente ont résidé en maison d'hébergement où elles ont profité de plusieurs services (suivi psychosocial, rencontres de groupes, référence, etc.). De manière générale, elles expriment une grande reconnaissance pour l'accueil, les services qui leur ont été offerts et le sentiment de sécurité qu'elles y ont éprouvé. Une répondante souligne que les maisons d'hébergement ont d'autant plus leur raison d'être pour des femmes comme elle dont la famille est éloignée et qui ne dispose que d'un faible réseau social. Deux répondantes ont apprécié le fait qu'il est possible dans leur maison d'avoir de la viande halal, de faire elles-mêmes la cuisine et même d'obtenir un tapis de prière.

Malgré leur grande appréciation, toutes ont fait allusion à la durée de séjour maximum. Elles soutiennent que la durée généralement permise est trop courte, surtout pour des nouvelles arrivantes. La durée leur semble d'autant plus courte lorsqu'elles considèrent les difficultés qu'elles ont eues à trouver un avocat pour les représenter devant la Commission de l'immigration et du statut de réfugié du Canada (CISR).

\section{Les services juridiques : "C'était difficile de trouver un avocat qui prend les dossiers d'immigration. "}

Toutes les femmes ont eu recours aux services d'un avocat pour leur divorce et, dans le cas des trois répondantes parrainées par leur conjoint et nouvellement arrivées au Canada, elles ont aussi eu besoin d'un avocat pour les représenter devant la CISR. 
Elles ont en commun le fait d'avoir éprouvé de la difficulté à trouver un avocat qui accepte des dossiers d'immigration. De plus, les récits des femmes rendent compte du sentiment de confusion qui les habite à propos, d'une part, des étapes suivant le dépôt d'une plainte à la police pour la violence physique subie et, d'autre part, du déroulement d'une audience devant la CISR, particulièrement concernant le risque d'être renvoyée dans leur pays d'origine.

\section{Le conjoint : une ressource?}

Peut-on citer les conjoints parmi les ressources que les répondantes ont mobilisées, ou du moins tenté de mobiliser, pour faire face à leur situation alors que ces derniers leur faisaient subir de la violence, les empêchaient d'accéder à des ressources ou contrôlaient leurs allées et venues? Nous soulevons la question puisqu'avant même leur arrivée au Canada, les répondantes voyaient leurs conjoints comme une ressource leur permettant d'obtenir la résidence permanente et ainsi de vivre "ailleurs ", cet ailleurs qui inspirait Nora et Hakima qui souhaitaient s'émanciper et se réaliser professionnellement, cet ailleurs où Hayat voulait voir son enfant bénéficier d'une bonne éducation.

Amira, Nora et Hakima voyaient également leurs conjoints comme une ressource sur laquelle compter pour s'intégrer dans leur nouveau pays de résidence, que ce soit pour les accompagner dans des démarches d'insertion socioprofessionnelle, les présenter à des amis, leur fournir le nécessaire pour se déplacer ou se divertir.

Certes, les conjoints n'ont pas répondu aux attentes des femmes. Cependant, tout est-il attribuable à une volonté de

"Comment considérer

l'impact des facteurs

structurels...

sans pour autant

déresponsabiliser les

conjoints des actes

de violence qu'ils

perpétuent?" leur part de contrôler leur partenaire? Comment considérer l'impact des facteurs structurels (déqualification professionnelle, difficultés d'insertion en emploi, chômage, discrimination et racisme à l'encontre des personnes arabo-musulmanes), sans pour autant déresponsabiliser les conjoints des actes de violence qu'ils perpétuent? La question est soulevée ici puisque les données de l'étude portent à croire que les conjoints de deux répondantes étaient confrontés à des difficultés d'insertion en emploi. Bien que professionnels de formation, l'un était sans emploi tandis que 
l'autre occupait un emploi de nuit. Ces deux conjoints ont évoqué des difficultés financières pour que les répondantes leur remettent le peu d'argent qu'elles possédaient à leur arrivée au Canada.

Ces difficultés d'ordre économique peuvent-elles s'être répercutées sur la dynamique du couple au sens où les conjoints n'étaient pas dans les meilleures conditions financières et morales pour répondre aux besoins des répondantes qui devaient compter sur eux pour les accompagner dans leur processus d'intégration? On peut penser ici à cette répondante dont les sorties de couple se limitaient à des promenades dans les centres d'achat ou encore à cette autre dont le conjoint ne lui fournissait pas l'argent dont elle avait besoin pour utiliser les transports en commun et se rendre aux ateliers d'intégration. Cette dernière ne parvenait pas non plus à obtenir de son époux l'achat d'articles nécessaires dans la maison. Ces exemples illustrent qu'il peut parfois être complexe de faire la distinction entre ce qui relève d'une volonté du conjoint de contrôler sa partenaire versus une incapacité réelle de lui offrir de meilleures conditions de vie.

\section{La maison : un espace à l'abri du racisme?}

La littérature suggère que le climat anti-arabo-musulman peut dissuader certaines femmes d'utiliser les services d'aide. Cela, parce qu'elles craignent de renforcer les stéréotypes négatifs à l'encontre des membres de leur groupe d'appartenance (Bendriss, 2007) ou encore parce qu'elles souhaitent protéger leur famille (Abu-Ras, 2007). Plus généralement, cela rejoint l'idée de Crenshaw (2005) à savoir que «[d]ans bien des cas, les femmes de couleur ont d'autant plus de mal à demander protection contre les violences familiales qu'elles désirent justement protéger ce havre que représente la maison contre les agressions du monde extérieur» (p. 67).

Cependant, aucune des répondantes de l'étude n'a exprimé avoir été dissuadée d'utiliser les services d'aide pour cette même raison. Ainsi, la maison ne peut être citée comme une ressource où les femmes se sont réfugiées ou qu'elles souhaitaient protéger. Il est toutefois intéressant de souligner que les trois répondantes qui sont de la même origine et de la même religion que leurs 
«Du fait des représentations sociales par lesquelles sont catégorisées les femmes arabes et musulmanes de manière dominante dans l'espace sociétal québécois, il était attendu que l'épouse d'origine arabe et d'appartenance musulmane soit soumise, résignée et docile..." conjoints se sont dites effectivement à l'abri du racisme lorsqu'elles étaient à la maison. Ce qui contraste nettement avec le cas d'Amira qui, elle, subissait le racisme dans la sphère domestique de la part de son conjoint et de sa belle-famille appartenant au groupe majoritaire. Du fait des représentations sociales par lesquelles sont catégorisées les femmes arabes et musulmanes de manière dominante dans l'espace sociétal québécois, il était attendu que l'épouse d'origine arabe et d'appartenance musulmane soit soumise, résignée et docile :

«Ça,je l'oublierai jamais... Il me disait :"Tu sais quoi Amira, ici c'est moi l'homme, c'est moi qui commande." Il me disait :"Chez vous [...] vous êtes des femmes soumises alors ici moi je suis dans mon pays, c'est moi qui commande". [...] Il disait :"Vous les Arabes, vous êtes des menteurs, on vous apprend à mentir dès votre jeune âge, vous savez pas vivre, vous avez pas beaucoup de respect pour les femmes." Et il me disait : "Regarde tout le mal qui se passe dans le monde" [...] Il insultait les Arabes : "Les femmes arabes vous êtes des femmes soumises, les Arabes c'est des menteurs..."J'étais comme oh... qu'est-ce que je suis en train de...» (Amira)

Comme le soutient Bendriss (2007, p. 10), «l'image fortement ancrée de la violence au sein du couple femme arabe et/ou musulmane et homme arabe et/ou musulman empêche de voir celle qui peut exister dans le couple femme arabe et/ou musulmane et homme québécois/canadien ". Or, le cas d'Amira montre que non seulement la violence conjugale peut exister dans un tel couple, mais aussi qu'elle peut prendre des formes de racisme.

Par ailleurs, aussi paradoxal que cela puisse paraitre, est-il possible que le contrôle exercé par les conjoints (par exemple, le contrôle sur les allées et venues, l'interdiction de sortir seule, l'interdiction de travailler) ait eu pour effet de "protéger " les répondantes des violences auxquelles sont exposées les 
femmes arabes et musulmanes dans l'espace public? Aucune des répondantes n'a exprimé avoir été victime pendant sa relation conjugale de telles formes de violence. La séparation semble toutefois avoir changé la donne. En l'occurrence, Amira qui occupe un emploi depuis sa séparation dit être la cible de commentaires à connotation raciste émis par ses collègues sur le ton de la plaisanterie. Aussi, on note que les secteurs d'emploi investis par les trois répondantes diplômées ne correspondent pas à leur champ de formation ni à leur expérience antérieure de travail. Il s'agit plutôt de secteurs d'emploi dans lesquels les personnes immigrantes sont surreprésentées : Hakima envisageait de suivre une formation pour travailler en garderie, Nora considérait la possibilité d'accepter un emploi en télémarketing et Amira travaillait dans le télémarketing en même temps qu'elle suivait une formation pour devenir infirmière auxiliaire. Enfin, Hayat, qui était en recherche d'emploi, a dit éprouver le sentiment qu'il était plus difficile pour elle de décrocher un emploi comparativement à d'autres femmes. Cependant, bien que cette dernière reconnaisse qu'il existe une discrimination à l'égard des personnes arabes et musulmanes, elle croit toutefois que dans son cas, c'est plutôt son âge (52 ans) et son manque d'expérience qui posent problème pour les employeurs.

Il est difficile, voire impossible, de démontrer que ces quelques expériences sont bel et bien des manifestations de la violence spécifiquement vécues par les femmes arabes et musulmanes dans l'espace public. La démonstration s'avère d'autant plus complexe dans le cas des répondantes puisqu'une partie de ces expériences (par exemple, la déqualification professionnelle et les difficultés d'intégration en emploi) pourrait être attribuable au fait qu'elles sont nouvellement arrivées au Canada $(n=3)$ et depuis peu en recherche d'emploi.

\section{La religion : source d'espoir et de résilience}

Les témoignages de Hayat, de Hakima et d'Amira à propos de la religion suggèrent qu'elle a été une source importante d'espoir et de résilience, et ce, tant pendant qu'après leur relation conjugale. Les résultats de notre étude rejoignent les observations d'Abugideiri 
(2007) qui soutient que plusieurs femmes musulmanes qui ont été victimes de violence conjugale et auprès de qui elle est intervenue identifient la foi comme la seule chose qui leur a permis de tenir puisqu'elles s'en remettaient à Dieu pour veiller sur elles (p. 111). Amira déclare : «si je m'en suis sortie, c'est grâce à Dieu.» Hakima raconte qu'il lui arrivait pendant sa relation de prier " pour qu'il n'arrive rien ce soir-là ". Après sa séparation, elle dit avoir lu régulièrement le coran pour se calmer, s'endormir et ne pas penser à son conjoint. Hayat pour sa part s'est servie de repères religieux tant pour confronter son conjoint et lui faire entendre que ses comportements violents étaient interdits dans l'islam que pour trouver une sérénité lorsqu'elle cherchait une issue à la violence qu'elle vivait. Elle a également consulté l'imam de la mosquée qu'elle fréquentait afin d'obtenir des conseils. Celui-ci lui a proposé d'intervenir auprès de son conjoint, mais ce dernier a refusé de le rencontrer. Plus tard, l'imam a dit à Hayat que le divorce était une option acceptable dans son cas et l'a encouragée dans ce sens en lui disant : "C'est le Bon Dieu qui t'a ouvert les yeux, c'est le Bon Dieu qui t'a ouvert les portes. " Ainsi, bien qu'elle souhaitait initialement éviter de divorcer, Hayat assume aujourd'hui sereinement et sans culpabilité sa décision de l'avoir fait. Plus généralement, elle dit que la religion l'a aidée à traverser les périodes où elle a souffert d'insomnie tout comme elle l'aide actuellement à persévérer dans sa recherche d'emploi, et ce, malgré les difficultés qu'elle rencontre.

Abugideiri (2007) avance que la foi permet aux femmes victimes de violence conjugale de croire que le conjoint sera tenu responsable de ses gestes. En éprouvant le sentiment que "...la religion a été source d'espoir et de résilience pour la plupart des répondantes. Leurs pratiques religieuses régulières leur ont procuré un sentiment de stabilité." justice sera faite, elles peuvent alors trouver une certaine sérénité, une stabilité émotionnelle et psychologique. Enfin, cela facilite le pardon comme l'exprime Hayat qui n'éprouve pas de rancune contre son conjoint dont elle remet le sort entre les mains de Dieu et à qui elle souhaite de trouver le droit chemin.

C'est en regard de tous ses éléments que nous suggérons que la religion a été source d'espoir et de résilience pour la plupart des répondantes. Leurs pratiques religieuses régulières leur ont procuré un sentiment de stabilité. L'ensemble de leurs croyances 
"Nous appréhendons ici les femmes comme des actrices de leur vie, et ce, même lorsqu'elles ont adopté des stratégies dites "passives »."

"À travers les stratégies qu'elles mettaient en cuvre pour éviter la violence, la faire cesser ou la fuir, elles anticipaient l'issue possible de leur situation en se référant aux savoirs cumulés au fil de leur expérience. » s'est avéré être un cadre de référence tant pour se rendre à l'évidence que les comportements violents de leur conjoint étaient inacceptables que pour se sécuriser face à ce qu'il adviendrait d'elles et de ce dernier.

\section{Les femmes elles-mêmes : une ressource}

Nous ne pourrions terminer cette partie sur les ressources sans faire référence aux femmes elles-mêmes qui se sont mobilisées et qui ont mis en place des stratégies pour faire face à leur situation au gré des circonstances. Nous appréhendons ici les femmes comme des actrices de leur vie, et ce, même lorsqu'elles ont adopté des stratégies dites "passives ». Des actrices qui ont pris des décisions en fonction de leurs expériences et des savoirs qui en sont issus.

La trajectoire expérientielle des répondantes depuis leur engagement conjugal et leur arrivée au Canada jusqu'à leur séparation leur a en effet permis de développer de nombreux savoirs. On peut penser ici à Hayat qui savait ce qui contrariait son conjoint et pouvait dégénérer en épisode de violence. De même, on peut penser aux savoirs liés à la religion qu'elle a utilisés pour confronter ce dernier pour qu'il cesse d'être violent à son encontre.

On peut également citer le cas de Nora et celui de Hakima qui ont rapidement mobilisé des ressources pour connaitre leurs droits en tant que nouvelles arrivantes et s'informer de l'aide qu'elles pourraient solliciter advenant qu'elles quittent leur conjoint. Enfin, Amira qui est arrivée au Québec sous un statut de résidence temporaire savait que ses possibilités étaient limitées tant qu'elle n'avait pas obtenu la résidence permanente. Ainsi, c'est en fonction des informations qu'elles avaient recueillies que Nora, Hakima et Amira ont pris la décision de se séparer ou pas.

En bref, les résultats de notre étude révèlent que les femmes ont été de véritables actrices sociales. À travers les stratégies qu'elles mettaient en œuvre pour éviter la violence, la faire cesser ou la fuir, elles anticipaient l'issue possible de leur situation en se référant aux savoirs cumulés au fil de leur expérience. Également, elles s'informaient des ressources disponibles et géraient stratégiquement celles qu'elles avaient déjà en leur disposition. 


\section{Conclusion}

Par notre recherche, qui se veut être exploratoire, nous avons tenté de mettre en relief certaines des ressources que les femmes arabes ou musulmanes mobilisent pour faire face la violence conjugale. À ce sujet, les résultats de notre recherche montrent que les femmes rencontrées n'étaient pas des victimes passives face à la violence conjugale qu'elles subissaient. Elles étaient des actrices à part entière qui ont su mobiliser de multiples ressources pour faire face à leur situation et c'est précisément la méthodologie employée dans le cadre de cette recherche qui a permis d'en rendre compte.

En effet, ces femmes ont été invitées à raconter leur histoire depuis leur engagement conjugal jusqu'à la séparation du couple. En nous inspirant de la méthode de récit de vie, nous cherchions alors à saisir "par quels mécanismes et processus des sujets en sont venus à se retrouver dans une situation donnée, et comment ils s'efforcent de gérer cette situation" (Bertaux, 1997, p.15). En employant la méthode de récit de vie, nous avons pu découvrir, entre autres, le contexte dans lequel s'inscrit l'expérience des femmes et les ressources qu'elles mobilisent pour faire face à leur situation. Il s'agit d'une méthode qui gagnerait à être utilisée davantage en intervention, car en donnant la parole aux femmes pour qu'elles se racontent elles-mêmes, non seulement avons-nous pu mieux comprendre leur situation, mais nous leur avons aussi permis de nous faire connaitre les multiples ressources qu'elles ont su mobiliser.

Les informations recueillies à propos des ressources ont permis de démontrer que ces dernières incluent les services d'aide en violence conjugale sans toutefois s'y limiter. D'autres ressources, dont la religion et les réseaux familiaux transnationaux, ont été évaluées comme étant des sources importantes de soutien tandis que les organismes offrant des services d'aide à l'emploi se sont avérés être des tremplins pour fournir une aide spécifiquement adaptée à la violence.

En ce qui concerne la religion, Rachédi et Legault (2008) soulignent que la personne intervenante peut avoir elle-même 
pris ses distances de ses appartenances religieuses et ainsi avoir parfois de la difficulté à accorder aux croyances de l'autre toute l'importance qu'elles méritent (p.126). Cela est d'autant plus vrai dans le contexte où l'islam est généralement vu comme une religion asservissante pour les femmes. Pourtant, comme le souligne Bendriss $(2007$, p. 6) à propos de la religion : « la croyance en Dieu procure un sens aux personnes qui y sont attachées, elle leur permet un équilibre psychique, elle leur donne des balises et des repères sociaux et symboliques, elle leur procure la paix, la sérénité et une force intérieure.»

Ainsi, le rapport des femmes avec leur religion gagnerait à être considéré pour être mis à contribution dans le cadre d'une intervention. Par ailleurs, Rachédi et Legault (2008) de même que Bendriss (2007) notent que la religion permet aux personnes de développer un sentiment d'appartenance à une communauté, de créer des liens et de les maintenir.Ainsi, le fait d'explorer le rapport qu'entretient une femme avec la religion peut également fournir aux intervenantes ou intervenants des informations à propos du réseau social de la femme, soit des possibilités d'entraide et de travail en partenariat.

Quant aux réseaux familiaux transnationaux, cette recherche a permis de démontrer qu'ils étaient une source importante de soutien pour les participantes. Il s'agit de réseaux avec lesquels les femmes maintiennent des contacts réguliers par l'entremise des technologies de communications telles que Skype. Il s'agit de technologies qui pourraient être mises à contribution en intervention pour travailler en partenariat avec la famille résidant dans le pays d'origine. En effet, certaines familles des répondantes craignaient que la femme se retrouve à la rue ou qu'elle soit renvoyée dans son pays d'origine si elle se séparait de son conjoint. Dans le cadre d'un travail en partenariat avec la famille et en utilisant ces technologies, les intervenantes et intervenants pourraient, entre autres, entendre les préoccupations des familles et de la femme, répondre à leurs questions, les informer quant à leurs droits et à l'aide disponible, élaborer avec elles un plan d'intervention ou mettre en place un scénario de protection. 
Les organismes offrant des services d'aide en employabilité comptent parmi les ressources que toutes les participantes à l'étude ont mobilisées à un moment ou l'autre de leurs trajectoires. Les participantes qui se sont tournées vers ces organismes avant de se séparer ne l'ont pas fait dans le but d'obtenir une aide spécifique quant à leur situation de violence conjugale; elles ont d'abord formulé une demande générale concernant leurs droits ou encore les démarches à suivre pour travailler. Non seulement cela indique que les besoins des femmes victimes de violence sont divers, mais aussi que les voies d'accès vers les services spécifiques en violence conjugale le sont tout autant. Dans cette perspective, le travail en partenariat avec d'autres organismes que ceux offrant des services spécialisés en violence conjugale gagnerait à être étendu.

"Les conclusions de cette recherche sont un appel à élargir notre conception des expériences de violence conjugale, et ce, en tenant compte des multiples formes de violence auxquelles les femmes sont exposées, aussi bien dans la sphère publique que privée. "
Les conclusions de cette recherche sont un appel à élargir notre conception des expériences de violence conjugale, et ce, en tenant compte des multiples formes de violence auxquelles les femmes sont exposées, aussi bien dans la sphère publique que privée. Les inégalités sociales et économiques qui affectent les communautés arabes et musulmanes, et de manière plus importante les femmes qui en sont issues, sont parmi les manifestations les plus évidentes de ces formes de violence. Prendre en compte ces formes de violence ne devrait pas pour autant nous amener à poser un regard victimisant sur ce groupe de femmes. Les conclusions de cette recherche sont plutôt un appel à appréhender les femmes comme des actrices sociales et à reconnaitre leurs forces, leurs savoirs expérientiels, leur réseau de soutien et l'ensemble des ressources dont elles disposent déjà et sur lesquelles on peut construire dans un contexte d'intervention.

Élargir notre conception des expériences de violence conjugale implique aussi de "porter un regard sans complaisance sur notre propre violence chaque fois que nous abordons des situations de violence " (Rousseau, 2002, p. 24). Ce qui ne saurait se faire sans que nous prenions conscience des stéréotypes et des préjugés dont nous sommes nous-mêmes porteuses ou porteurs. Cela ne saurait se faire non plus sans que nous nous questionnions sur les pratiques d'intervention que nous privilégions et plus généralement sur les réponses sociales à la violence. Quels sont 
leurs effets sur la valeur que nous accordons à l'analyse que les femmes font de leur situation, aux stratégies qu'elles mettent en œuvre, aux ressources qu'elles mobilisent et aux solutions qu'elles privilégient pour enrayer la violence de leur vie?

Comme nous l'avons souligné, aucune femme n'est restée passive face à la violence qu'elle subissait. Toutes souhaitaient que cette violence cesse et toutes ont considéré dans ce sens diverses pistes de solution en mobilisant des ressources, et ce, dans divers espaces : au parc, via le web, aux ateliers d'intégration pour les nouveaux arrivants, dans des organismes d'aide à l'intégration des immigrants, dans des centres de femmes, à la clinique ou à la mosquée. Dans ces espaces, certaines ont exploré des solutions comme celle de faire intervenir l'imam ou le père pour qu'il parle au conjoint. Ce qui soulève la question des alternatives offertes aux femmes qui souhaitent que la violence cesse : les choix des femmes victimes de violence consistent-ils essentiellement à accepter la violence de leur conjoint ou à se séparer de lui? Cette dernière question est d'autant plus complexe que les résultats de la recherche suggèrent que la séparation n'a pas nécessairement mis fin à la violence. Non seulement la violence conjugale n'a pas cessé après la séparation (par exemple, une plainte déposée à la CISR), mais aussi c'est à partir de la séparation du couple que les répondantes ont davantage été exposées aux formes de violence spécifiquement vécues par les femmes arabes et musulmanes dans l'espace public. Si tel est le cas, le " choix » des femmes arabes et musulmanes, lorsqu'elles sont victimes de violence conjugale, consiste-t-il alors à choisir le moindre mal, soit entre la violence à laquelle elles sont exposées dans l'espace public et celle qu'elles subissent dans la sphère privée? Comme nous pouvons le constater, certains résultats de notre étude font émerger de nouvelles pistes de recherche qui méritent d'être explorées dans de futures études.

\section{Notes}

Sous la direction d'Elizabeth Harper et de Lilyane Rachédi, professeures à l'École de travail social, UQAM. 
2 Cette étude a été réalisée grâce au soutien financier du CRSH et du FQRSC.

3 Ces contrôles ont pris les formes suivantes : ne pas fournir ou limiter le nombre de cartes d'appel dont la femme a besoin pour téléphoner à sa famille; prétendre ne pas avoir l'équipement technique nécessaire pour communiquer par internet (Skype), contrairement à ce qui avait été annoncé avant l'arrivée de la femme au Canada; lire les courriels pour vérifier ce que la femme confie à sa famille; menacer la femme de la battre ou de la renvoyer au pays si elle parle de la violence à sa famille, etc.

\section{Bibliographie}

ABUGIDEIRI, Salma Elkadi (2007). «Domestic violence among Muslims seeking mental health counselling ", dans Maha B. Alkhateeb et Salma Elkadi Abugideiri (dires.), Change from within: Diverse perspectives on domestic violence in Muslim communities, Great Falls, Peaceful Families Project, p. 91-116.

ABU-RAS, Wahiba (2007). "Cultural beliefs and service utilization by battered Arab immigrant women ", Violence Against Women, Vol. 13, № 10, p. 1002-1028.

ALKHATEEB, Maha B., et Salma ABUGIDEIRI (2007). «Introduction », dans Maha B. Alkhateeb et Salma Elkadi Abugideiri (dires.), Change from within: Diverse perspectives on domestic violence in Muslim communities, Great Falls, Peaceful Families Project, p. 13-27.

BAOBAID, Mohammed (2002). Access to Women Abuse Services by Arab-speaking Muslim Women in London, Ontario: Background Investigation and Recommendations for Further Research and Community Outreach, [rapport de recherche], London, Ontario, Centre for research on violence against women and children, $27 \mathrm{p}$.

BENDRISS, Naïma (2005). Représentations sociales, ethnicité et stratégies identitaires: le cas des femmes arabes $d u$ Québec, [thèse de doctorat], Montréal, Université de Montréal, 501 p.

BENDRISS, Naïma (2007). Enjeux pour la pratique dans un contexte professionnel. La prise en compte de la diversité religieuse dans l'offre de services en violence conjugale, [Actes de la journée d'étude du 27 novembre 2007], Montréal, Table de concertation en violence conjugale de Montréal, 12 p.

BENDRISS, Naïma (2009). "Les représentations sociales des Québécoises d'origine arabe : quels impacts dans la société en général et sur le marché du travail en particulier? ", dans Micheline Labelle et Frank W. Remiggi (dirs.), Inégalités, racisme et discriminations : Regards critiques et considérations empiriques, Les cahiers de la CRIEC, No 33, p. 59-76.

BERTAUX, Daniel (1997). Les récits de vie : Perspective ethnosociologique, Paris, Nathan, 128 p.

BOUCHARD, Gérard, et Charles TAYLOR (2008). Fonder l'avenir. Le temps de la conciliation, Rapport de la Commission de consultation sur les pratiques d'accommodement reliées aux différences culturelles, Québec, 307 p.

BURMAN, Erica, et Khatidja CHANTLER (2005). "Domestic violence and minoritisation: Legal and policy barriers facing minoritized women leaving violent relationships ", International Journal of Law and Psychiatry, Vol. 28, p. 59-74.

CHOUAKRI,Yasmina (2003). « Sexisme et racisme : La discrimination et les obstacles à l'intégration des femmes des communautés arabes et musulmanes ", réf. du 10 avril 2015, http://www.tgfm.org/mtl/ files/chouakri.pdf. 
CÔTÉ, Andrée, Michèle KÉRISIT et Marie-Louise CÔTÉ (2001). Qui prend pays... L'impact du parrainage sur les droits à l'égalité des femmes immigrantes, [rapport de recherche], Ottawa, Condition féminine Canada, $252 \mathrm{p}$.

CRENSHAW, Kimberle Williams (2005). «Cartographie des marges : intersectionnalité, politique de l'identité et violence contre les femmes de couleur ", Cahiers du genre, № 39, p. 51-82.

HAJ-YAHIA, Muhammad (2002). "Attitudes of Arab women toward different patterns of coping with wife abuse ", Journal of Interpersonal Violence, Vol. 17, $\mathrm{N}^{\circ}$ 7, p. 721-745.

HAJ-YAHIA, Muhammad, et Elisheva SADAN (2008). « Issues in intervention with battered women in collectivist societies ", Journal of Marital and Family Therapy,Vol. 34, № 1, p. 1-13.

HAMDANI, Daood (2004). Muslim women: Beyond perceptions, [rapport de recherche], Toronto, Canadian Council of Muslim Women.

HAMDANI, Daood (2006). Engaging Muslim women: Issues and needs, [rapport de recherche], Toronto, Canadian Council of Muslim Women.

HOOKS, bell (1984). Feminist theory from margin to center, Boston, South End Press, 179 p.

OSMANI, Farida (2007). Rêves brisés : typologies des violences faites aux femmes arabes et aux femmes musulmanes : enquête exploratoire à Montréal, [rapport de recherche], Montréal, Fédération des femmes du Québec, 91 p.

RACHÉDI, Lilyane, et Gisèle LEGAULT (2008). " Le modèle interculturel systémique ", dans Gisèle Legault et Lilyane Rachédi (dires.), L'intervention interculturelle, Montréal, Gaëtan Morin Éditeur, p. 121-142.

ROUSSEAU, Cécile (2002). "Repenser la vulnérabilité chez les femmes immigrantes et réfugiées ", dans Jacqueline Oxman-Martinez et Nicole LapierreVincent (dires.), Statut précaire d'immigration, dépendance et vulnérabilité des femmes à la violence : les impacts sur leur santé, Actes du séminaire, Montréal, Centre d'études appliquées sur la famille, p. 18-25.

ROY, Ghislaine (2008). "Les outils de pratique ", dans Gisèle Legault et Lilyane Rachédi (dires.), L'intervention interculturelle, $2^{\mathrm{e}}$ éd., Montréal, Gaëtan Morin Éditeur, p. 167-195.

SARIS, Anne, et collab. (2007). Étude de cas auprès de Canadiennes musulmanes et d'intervenants civils et religieux en résolution de conflits familiaux : une recherche exploratoire menée à Montréal en 2005-2007, réf. du 10 avril 2015, http://edoc.bibliothek.uni-halle.de/servlets/MCRFileNodeServlet/ HALCoRe_derivate_00002510/Étude\%20de\%20cas\%20auprès\%20de\%20Canadiennes $\% 20$ musulmanes.pdf;jessionid $=$ jnax53qhqih?hosts $=$

STATISTIQUE CANADA (2007). Profils de communautés ethniques du Canada : La communauté arabe au Canada, 89-621-XIF, Ottawa, Statistique Canada, Division de la statistique sociale et autochtone.

TAÏBI, Bouchra (2013). Violence conjugale et ressources mobilisées : une analyse intersectionnelle d'expériences de femmes arabo-musulmanes au Québec, [mémoire de maîtrise], Montréal, Université du Québec à Montréal, $162 \mathrm{p}$.

VASHTI PERSAD, Judy, et Salome LUKAS (2002). "No hijab is permitted here": A study on the experiences of muslim women wearing hijab applying for work in the manufacturing, sales and service sectors, [rapport de recherche], Toronto, Women Working With Immigrant Women.

WEST, Carolyn M. (2005). «Domestic violence in ethnically and racially diverse families:The "political gag order" has been lifted", dans Natalie J. Sokoloff (dirs.), Domestic violence at the margins: A reader at the Intersections of Race, Class, and Gender, New Jersey, Rutgers University Press, p. 157-173. 